\title{
Discussion
}

\section{Alzheimer Research Forum Live Discussion: Imaging in Alzheimer's Disease: The Current State of Affairs ${ }^{1}$}

Live Discussion led by Bill Klunk, Julie Price, Chet Mathis, Eric Reiman, and Clifford Jack on 6 May 2005.

http://www.alzforum.org/res/for/journal/klunk/default.asp

Participants: Bill Klunk (University of Pittsburgh), Julie Price (University of Pittsburgh), Chet Mathis (University of Pittsburgh PET Facility), Eric Reiman (University of Arizona), Clifford Jack (Mayo Clinic College of Medicine), Tom Fagan (Alzforum), June Kinoshita (Alzforum), Benedict Albensi (Washington University), Betty Tuller (Brigham and Women's Hospital), Hank F. Kung (University of Pennsylvania), Jessica Andrews (Washington University), Keith Johnson (Harvard University), Mikolaj A. Pawlak (Poznan University of Medical Sciences), Nick Fox (Dementia Research Centre, United Kingdom), Reisa Sperling (Brigham and Women's Hospital), Edward Zamrini (University of Alabama at Birmingham, Memory Disorders Clinic).

Tom Fagan: I am Tom Fagan and I will be moderating our chat today. Chet, do you want to start us off with a topic?

Chet Mathis: Hank, where does SPECT amyloid imaging in humans stand?

Hank F. Kung: We are doing I-123 IMPY now [IMPY, or 6-iodo-2-(4'-dimethylamino-)phenyl-imidazo[1,2a]pyridine, is a thioflavin derivative that has binding affinity for amyloid- $\beta(\mathrm{A} \beta)$ in solution and in postmortem Alzheimer disease (AD) samples] [1]. The data look good but we are not ready to publish yet. Bill, what is the status of your Pittsburgh compound B (PIB) study in AD patients?

Bill Klunk: We have done about 30 subjects.

\footnotetext{
${ }^{1}$ Note: The transcript has been edited for clarity and accuracy.
}

Hank F. Kung: Mike Welch told me that Washington University has done 70 patients.

Reisa Sperling: How about some discussion of the anatomic distribution of PIB retention and its relationship to hippocampal atrophy/dysfunction?

Edward Zamrini: Bill, while amyloid pathology is most evident in the parietotemporal areas, PIB seems to concentrate in the frontal lobes more. Any new thoughts on that?

Bill Klunk: Actually, the latest data show [PIB binding] to be highest in the precuneus, with parietal second and frontal a close third.

Clifford Jack: Bill, this is the first I have heard that frontal cortex is not most prominent. Is this a new finding? 
Chet Mathis: Cliff, the frontal cortex has a lower concentration than do the other two areas, but looks most prominent in the scans because it is a larger volume.

Edward Zamrini: Is there a differential effect of localization of PIB according to stage of disease? I know it may be too early to tell.

Mikolaj Pawlak: How about PET-PIB imaging for mild cognitive impairment/amnestic mild cognitive impairment $(\mathrm{MCI})$ ? Are there any differences between those two groups?

Bill Klunk: Disease-stage studies are just beginning, but we know if there is a lot already there by the MCI stage. We have not done enough MCI versus MCI amnestic to know if there is a difference, at least in Pittsburgh.

Eric Reiman: Bill, the pattern of PIB deposition nicely mirrors the pattern of hypometabolism and hypoperfusion (e.g., precuneus $>$ parietal $>$ frontal). Any chance the PIB measurements are influenced by blood flow (radiotracer delivery)?

Bill Klunk: We have blood flow correlation studies underway, but kinetic modeling indicates PIB retention is independent of blood flow.

Hank F. Kung: Bill, what do you mean by independent of blood flow?

Bill Klunk: I mean, there is not a strong association between the initial rate of uptake of PIB and late-time distribution in $\mathrm{AD}$ subjects.

Hank F. Kung: Chet, are you ready to test your fluorine-PIB at the University of Pittsburgh?

Chet Mathis: Hank, there are F-18 PIB compounds being used in human studies at Turku, Finland, and we are waiting to see which are the best.

Betty Tuller: Bill and Chet, can you give us a comparison of the F-18 versus the PIB compound in relative efficiency for detecting $\mathrm{A} \beta$ in patient populations?

Chet Mathis: Betty, actually it is too early to say much about the F-18 versus the PIBs.

Benedict Albensi: All, Higuchi et al. [2] just published a paper on $19 \mathrm{~F}$ and $1 \mathrm{H}$ MRI detection of $\mathrm{A} \beta$ plaques.
Mikolaj Pawlak: How about amyloid PIB imaging in mixed neurodegenerative disorders like Lewy body dementia (LBD)?

Bill Klunk: Chris Rowe in Melbourne has done studies and will present in Toronto; data from Lewy body dementia patients are similar to those from AD patients in very general terms.

Betty Tuller: Bill, what is your opinion of the current status of the F-18-FDDNP versus PIB?

Chet Mathis: Betty, FDDNP gives a much lower signal than PIB (about fivefold lower).

Keith Johnson: Bill, Chet, Julie, we have now done 13 subjects at Massachusetts General Hospital (MGH); are you using $15 \mathrm{mCi}$ and image for 45 minutes, or do you think it can be fewer Curies and shorter exposure?

Julie Price: Keith, the measure is more stable from 40 to 90 minutes.

Bill Klunk: Fifteen $\mathrm{mCi}$ of PIB is only 0.25 REM whole-body exposure, so there is little reason to want to reduce it. The yearly limit is 5 REM.

Betty Tuller: Bill and Chet, what is the maximum time between synthesizing the tracer and injection to ensure a decent PET image?

Chet Mathis: Betty, the stability of the tracer is good for 60 minutes, and as long as there is $10-15 \mathrm{mCi}$ for injection and a minimum specific activity of 300 $\mathrm{Ci} /$ millimole (at Pittsburgh), it should be fine.

Julie Price: Keith, are you scanning 45 to 90 or 0 to 45 minutes?

Keith Johnson: Do you routinely acquire from 0 to 90 , or do you start at 40 minutes? We are doing 0 to 90.

Julie Price: Yes, 0 to 90, for sure.

June Kinoshita: Addendum to Mikolaj's question: What about functional imaging correlated with PIB?

Mikolaj Pawlak: How about correlation between magnetic resonance spectroscopy (MRS) of medial temporal lobe (MTL) and PIB concentration? 
Bill Klunk: Mikolaj, we have not looked at MRS versus PIB at this point. We are waiting for Keith and Reisa to do that.

Reisa Sperling: We are working on it.

Mikolaj Pawlak: How about PIB and MTL atrophy dynamics over time?

Bill Klunk: Mikolaj, we are currently gathering MR structural data on all PIB subjects and will eventually start the atrophy-amyloid correlation analyses.

June Kinoshita: There is a great deal of emphasis on the MTL, but at the Bar Harbor workshop last summer, Bill, you mentioned that some posterior cortical and retrosplenial regions may be affected early. Can you discuss that further?

Bill Klunk: June, the precuniform cortex seems to be one of the earliest affected regions, not only for PIB, but also fluorodeoxyglucose (FDG) and cortical atrophy. It plays a major role in processing limbic information.

Clifford Jack: Same with MRS, that is, the posterior cingulate (PC)-precuniform $(\mathrm{PCu})$ is affected early.

Nick Fox: Our serial MRI studies also support early involvement of posterior cingulate and precuneus, including in familial AD.

June Kinoshita: How do the changes in posterior cingulate and precuneus compare with MTL changes? Any sense of which is earlier, which is more sensitive or specific?

Bill Klunk: The MTL amyloid studies postmortem show this to be an area with about one-fifth the amyloid load of cortex. PIB shows a consistently higher MTL retention in $\mathrm{AD}$; the amplitude is much lower. We cannot yet really say what comes first until we follow amyloid deposition longitudinally.

Eric Reiman: June, we appear to see the posterior cingulate and precuneus change very early, for example, in young adult ApoE4 heterozygotes who may be at risk for $\mathrm{AD}$ dementia 40 to 50 years later. Generally, hippocampal atrophy is seen later, may parallel the earliest cognitive changes, and may herald the subsequent onset of dementia. As baboon and lesion studies have shown, the FDG PET changes could reflect the den- sity/activity of neuronal terminals projecting from the MTL.

June Kinoshita: Are the changes seen in PC and PCu in both the functional and structural realm?

Tom Fagan: Bill, naive question, but what is the status on getting PIB-PET or any other imaging approved for diagnostic purposes?

Bill Klunk: Tom, we need more extensive studies to justify this to the FDA. We just submitted our Investigational New Drug (IND) application this week.

Eric Reiman: In response to the diagnosis question: In September, the Centers for Medicare and Medicaid Services (CMS) approved FDG PET for limited use in the differential diagnosis of dementia. According to the CMS, patients should have dementia and cognitive decline for at least 6 months; clinicians should have completed a comprehensive medical evaluation; and there should be remaining uncertainty about the differential diagnosis between $\mathrm{AD}$ and frontotemporal dementia (FTD).

Tom Fagan: Bill, do you have any sense for what is the smallest change in amyloid load you can measure with PIB, percentagewise?

Julie Price: Tom, the intrasubject variability is 5 to 6 percent, and we have detected significant group differences of about 15 percent.

Mikolaj Pawlak: How does white matter lesion (WML) load influence PIB imaging results?

Betty Tuller: This is a general question for anyone who can answer. If you are performing an AD study, would you expect to see amyloid degradation within 3 or 6 months? Or is it only logical to assess degradation over yearly time courses?

Bill Klunk: Betty, I assume you mean with antiamyloid therapies. No one knows; it depends on the type of therapy, I would guess.

Clifford Jack: Bill and Chet, have you done any therapeutic studies in mice with PIB using Brad Hyman's cortical window approach?

Bill Klunk: Cliff, Bob Brendza and Dave Holtzman at Washington University have done similar studies [3]. 
Clifford Jack: What did they find?

Bill Klunk: They showed using the amyloid imaging fluorophore, methoxy-X04, that when plaques dissolve, dystrophic neurites seem to repair themselves.

Clifford Jack: Bill and Chet, maybe you guys have published this, but can you comment on test-retest stability of PIB on short-term serial studies?

Julie Price: The test-retest variability is about 5 to 6 percent; the paper is in press in J. Cerebral Blood Flow Metab. The average test-retest was 10 percent in areas dominated by nonspecific binding, 5 to 6 percent in high-binding areas.

Clifford Jack: Five percent is very good. Bill, is the PIB MTL finding a manifestation of spatial resolution of PET, that is, the same thing that is seen in FDG?

Chet Mathis: Cliff, the low PIB in MTL is consistent with postmortem studies. Our PET studies are atrophy corrected by MR. Spatial resolution/partial volume does not account for the very low concentration of PIB in MTL.

Clifford Jack: So PIB concentration in MTL matches amyloid burden postmortem, right?

Bill Klunk: Yes, in relation to the cortex. We are also further exploring the types of amyloid deposits (e.g., fibrillar vs. amorphous) that are best detected by PIB.

June Kinoshita: I would also like to toss out a question about a study from Nick Fox's group, just published in Neurology [4], about the cortical shrinkage that correlates with response to AN1792 (the Elan vaccine). What ideas do you all have to explain this counterintuitive observation? What imaging resources could be used to shed light on what is going on?

Eric Reiman: June, as Nick noted earlier, he is finding increased atrophy rates in this location relatively early in AD. In cross-sectional, voxel-based morphometry (VBM) comparisons, we also see decreased gray matter density in some of the same regions in which we see hypometabolism, though the PET changes are not solely attributable to atrophy and partial-volume averaging. As Mony de Leon has been showing, these VBM procedures may not be as good at looking at hippocampal gray matter atrophy, because of some spatial standardization challenges.
Nick Fox: As far as the AN1792 study is concerned, I still feel that the simplest explanation is that the volume change represents amyloid loss; of course, there may be multiple mechanisms at play.

Bill Klunk: Nick, I assume you mean amyloid plus surrounding water and tissue reaction, because there is not enough mass of amyloid to explain this by itself.

Clifford Jack: Another possibility is that those who mounted an antibody response also had encephalitis. Patients with florid encephalitis (and who would be expected to do worse clinically?) were excluded. Patients with a low level of encephalitis who later improved clinically due to amyloid reduction had shrinkage of the brain due to encephalitis.

Nick Fox: I think there are a number of experiments that can be done to help separate out some of the alternatives. We are in the midst of measuring cerebellar and pontine changes to check for a simple hydration change, which I do not believe.

June Kinoshita: I believe the responders had decreased cerebrospinal fluid (CSF) tau (phospho-tau?). Was there a similar correlation with CSF A $\beta 40 / 42$ ratios?

Hank F. Kung: Is there any postmortem data to support the reduction of $\mathrm{A} \beta$ ?

Bill Klunk: Hank, four studies have been reported; three show focal amyloid reduction. The fourth, done very early in the treatment course, shows microglia filled with amyloid.

Nick Fox: Bill, the question of the volume (as opposed to mass) is interesting. The autopsy studies using stereology suggest that amyloid accounts for up to 10 percent of some cortical regions. There is a relative paucity of data on the volume occupied by amyloid; one has to remember that plaques contain things other than pure amyloid.

Bill Klunk: Very true, Nick.

Eric Reiman: I am betting it is the removal of water along with amyloid loss rather than encephalitis that accounts for the reduction in brain volume.

Clifford Jack: Eric, you could be right, but I doubt that the encephalitis was an all-or-nothing phenomenon. I 
am sure there were varying grades of severity in the study population.

Nick Fox: Eric, I agree it is very difficult to exclude that possibility, but I do not see how encephalitis would explain the dissociation between volume loss and lack of cognitive deterioration. Also, I do not think that subclinical encephalitis can account for the changes. For a start, the baseline scans were done before dosing.

Clifford Jack: Nick, I am not a strong advocate, but threw out encephalitis as a possible idea. The idea is that some level of encephalitis is a requirement in order to see any amyloid reduction. The cognitive deterioration due to encephalitis in those with a large amyloid reduction effect would be overcome by the benefits to cognition of amyloid load reduction - it is an idea.

Nick Fox: Cliff, I think that mixture of effects is plausible.

Reisa Sperling: Nick, you also found reduction in hippocampal volumes (albeit less than global) which is not loaded with amyloid per PIB (and some postmortem studies). What do you think regarding MTL changes?

Nick Fox: What we really needed was a number of scans (as was planned), rather than just two, in order to assess the time course.

June Kinoshita: Could there be indirect effects that AN1792 has on microglial responses?

Nick Fox: June, yes, I think a contribution of reduced microglia is another possibility.

June Kinoshita: There is a new method for imaging microglial activation. I am waiting for Brad Hyman's group to apply that to their in vivo imaging system [5].

Jessica Andrews: Eric, what do you make of all the other metabolic reductions in young ApoE4 heterozygotes you and colleagues reported in your recent paper, for example, lateral parietal/frontal splotches? [6]

Eric Reiman: Jessica, this pattern of reductions has been reproduced following MTL lesions in baboons, raising the possibility that it reflects projections from that area. Still, these changes occur very early - before the development of plaque and all by an isolated tan- gle, raising the question about what underlying brain changes might participate in the subsequent development of AD histopathology.

Jessica Andrews: And apparently atrophy is present early on in these non-MTL areas, so it raises the question that MTL parietal projections might not account for all of the metabolic reductions.

Clifford Jack: Jessica, if you do VBM maps, you see the same reduction in brain volume with MRI that Eric first described in the posterior cingulate.

Eric Reiman: Jessica, perhaps reductions in parietal and PC gray matter density reflect loss of synapses (a dying back of neurons), too.

Nick Fox: Eric, could the ApoE4-related differences reflect a phenotypic difference - from a very early age?

Eric Reiman: Nick, our young adult findings raise more questions than answers. But, it is indeed possible that they develop earlier than young adulthood and are even congenital. What is interesting is that these reductions predict the locations of progressive cerebral glucose metabolism (CMRgl) decline and amyloid accumulation a few decades later.

June Kinoshita: I also want to ask you all to suggest a wish list or priority list for new studies using existing imaging technologies, and also for novel imaging targets that the field needs to elucidate early molecular mechanisms, and so on.

Bill Klunk: I would wish for a tangle-specific tracer.

June Kinoshita: Any progress there?

Bill Klunk: No, not in Pittsburgh. We have not tried anything along those lines.

June Kinoshita: I have wondered how readily one can get a tracer into neurons to label intraneuronal tau aggregates. Is that one of the challenges?

Bill Klunk: June, not really; once you cross the bloodbrain barrier (BBB), the rest should be downhill.

Nick Fox: I would wish for us to be able to image synapses, as well, but failing that, I would settle for studies in which we combine amyloid and tangle tracers 
with structural brain changes. I would also love to be able to visualize amyloid using structural MRI.

June Kinoshita: Nick, is functional imaging a reasonable surrogate for synaptic changes, or are there more specific molecular targets that you fantasize about?

Nick Fox: June, since we were asked for a wish list, I would still include markers of synaptic integrity in addition to the valuable information from functional imaging.

June Kinoshita: Nick, what would be your top candidates for markers of synaptic integrity?

Eric Reiman: June, whereas FDG PET provides some information about synaptic density, those measurements can be confounded by synaptic activity, glial density, and metabolism, and so on. Something like a synaptophysin marker (or some other synapse-specific receptor) would be great.

Tom Fagan: We have come to the end of our hour. Thanks to the Pittsburgh group and everyone.

\section{References}

[1] M.P. Kung, C. Hou, Z.P. Zhuang, B. Zhang, D. Skovronsky, J.Q. Trojanowski, V.M. Lee and H.F. Kung, IMPY: an improved thioflavin-T derivative for in vivo labeling of beta-amyloid plaques, Brain Res 956 (2002), 202-210.

[2] M. Higuchi, N. Iwata, Y. Matsuba, K. Sato, K. Sasamoto and T.C. Saido, $19 \mathrm{~F}$ and $1 \mathrm{H}$ MRI detection of amyloid beta plaques in vivo, Nat Neurosci 8 (2005), 527-533.

[3] R.P. Brendza, B.J. Bacskai, J.R. Cirrito, K.A. Simmons, J.M. Skoch, W.E. Klunk, C.A. Mathis, K.R. Bales, S.M. Paul, B.T. Hyman and D.M. Holtzman, Anti-A $\beta$ antibody treatment promotes the rapid recovery of amyloid-associated neuritic dystrophy in PDAPP transgenic mice, J Clin Invest 115 (2005), 428-433.

[4] N.C. Fox, R.S. Black, S. Gilman, M.N. Rossor, S.G. Griffith, L. Jenkins and M. Koller, AN1792(QS-21)-201 Study. Effects of $\mathrm{A} \beta$ immunization (AN1792) on MRI measures of cerebral volume in Alzheimer disease, Neurology 64 (2005), 1563-1572.

[5] A. Nimmerjahn, F. Kirchhoff and F. Helmchen, Resting microglial cells are highly dynamic surveillants of brain parenchyma in vivo, Sciencexpress (14 April 2005).

[6] R.J. Caselli, E.M. Reiman, D. Osborne, J.G. Hentz, L.C. Baxter, J.L. Hernandez and G.G. Alexander, Longitudinal changes in cognition and behavior in asymptomatic carriers of the APOE e4 allele, Neurology 62 (2004), 1990-1995. 